\title{
Phase Field Model for Solidification with Boundary Interface Interaction
}

\author{
Jie Liao \\ School of Science, East China University of Science and Technology \\ Shanghai, 200237, P. R. China \\ liaojie@ecust.edu.cn
}

Keywords: phase field model, boundary interface interaction, dynamic contact angle

\begin{abstract}
By incorporation the surface free energy in the free energy functional, a phase field model for solidification with boundary interface intersection is developed. In this model, the bulk equation is appropriately modified to account for the presence of heat diffusion inside the diffuse interface, and a relaxation boundary condition for the phase field variable is introduced to balance the interface energy and boundary surface energy in the multiphase contact region. The asymptotic analysis is applied on the phase field model to yield the free interface problem with dynamic contact point condition.
\end{abstract}

\section{Introduction}

We study the dynamics of interaction between interface and external boundary in solidification. Consider the motion of an interface curve $\Gamma(t)$ in a channel $\Omega=\{\mathbf{x}=(x, y): x \in \mathbb{R}, 0 \leq y \leq L\}$. The interface $\Gamma(t)$ intersects the boundary of $\Omega$ at two points on each side of the channel and divides the channel $\Omega$ into two bulk regions $\Omega_{+}(t)$ and $\Omega_{-}(t)$, where $\Omega_{+}(t)$ denotes the liquid region, $\Omega_{-}(t)$ denotes the solid region and $\Gamma(t)$ is the solid/liquid interface. The evolution of the interface is extensively studied when the interface is a closed curve that never intersects the external boundary. We will concern the dynamics of the boundary-interface interaction.

The motion of the interface is controlled by diffusion of a dimensionless scalar field $u(\mathbf{x}, t)$. For solidification of a pure material, $u$ is the temperature relative to the equilibrium freezing temperature scaled by latent heat of fusion and the heat capacity.

In the bulk phases, $u$ solves two separate diffusion equations

$$
u_{t}=k_{ \pm} \triangle u \text { in } \Omega_{ \pm}
$$

in which $k_{ \pm}$are the diffusion coefficients in liquid and in solid, respectively. For a solidification problem, the solutions to the above are joined at the solid-liquid interface $\Gamma(t)$, a free boundary of unknown shape and position. One might first assume the temperature $u$ to be continuous across $\Gamma(t)$. The advancing or retreating of the interface means the material is changing phase and releasing or absorbing latent heat of fusion, by conservation of energy, $\nabla u$ must satisfy the jump condition

$$
V=-[[k \partial u / \partial m]]_{-}^{+} \text {across } \Gamma,
$$

where $V$ is the normal velocity of $\Gamma$, positive when solid is advancing into liquid, $m$ is the unit normal on $\Gamma$ pointing to the liquid. $\left[[\cdot \cdot]_{-}^{+}\right.$is the jump of · across the interface.

Finally, $u$ must satisfy the Gibbs-Thomson condition

$$
u=-\delta \kappa-\tau V \text { on } \Gamma
$$

where $\kappa$ is the curvature of $\Gamma$, positive when the solid is convex. The material parameter $\delta$ is the capillary length and is proportional to the interfacial energy. The kinetic parameter $\tau>0$ measures the interfacial mobility by linear kinetic relation

$$
V=-M(u+\delta \kappa) \text { with } M=1 / \beta
$$


In this setting, we need to solve both the temperature field $u(\mathbf{x}, t)$ and the shape and position of the free boundary $\Gamma(t)$. The evolution of the interface $\Gamma(t)$ is governed by the above kinetic relation (4), this equation can be understood similarly to curvature flow equation, which is a second order parabolic type partial differential equation. This problem may be solved when the interface is a closed curve that never intersects the external boundary. When the free boundary $\Gamma(t)$ intersect the external boundary, to complete the formulation, we need to impose conditions on the boundary-interface contact points to incorporate the boundary-interface interaction in the evolution of the interface, thus additional dynamic conditions on the contact points are required[16].

One effective way for solving this problem is by phase field model, in which the sharp interface is replaced by a diffuse interface and an auxiliary order parameter $\varphi$, the phase field, is introduced to indicate the phase. The quantity $\varphi$ is a continuous variable that takes constant in each bulk phase, say 0 in the solid and 1 in the liquid, and increase from 0 to 1 over a thin layer, the diffuse interface. A system of phase field equations are constructed to govern the time evolution of both $\varphi$ and $u$, which incorporate the interfacial physics of the problem and the release of latent heat.

The standard phase-field model, a version of the Model C of Halperin, Hohenberg and Ma [7] was originally derived on the basis of a gradient functional for the free energy of the system, with dynamics that ensure the free energy decreases with time. The time rate of change of the order parameter is coupled to the energy equation to incorporate the release of latent heat as solidification occurs. This model was further elaborated in Caginalp [2], Fix [6] and Langer [11]. See also the recent reviews $[13,17]$. In the above mentioned works, however, the interaction of external boundary and interface was neglected. We will use the modified free energy functional proposed by Cahn[3] to include the boundary-interface contact energy. See also $[14,15]$. Thus a modified gradient flow with relaxational boundary condition were derived. We further illustrate by asymptotic analysis that the relaxational boundary condition leads to a dynamic contact angle relation at the boundary-interface intersection. This relaxational boundary condition and dynamic contact angle relation are more reasonable to study the boundary-interface interaction in phase transition dynamics than Neumann conditions proposed in [10] and stationary contact angle conditions proposed in [4], when the boundary-interface interaction is significant.

In next section, we will describe a phase-field model that resolves the above problem. Asymptotic analysis of this model and the leading order interface dynamics will be presented as followed.

\section{Phase field model}

We briefly indicate the construction of the phase field model for solidification of a pure material from its melt. For simplicity of presentation, we assume that all quantities are isotropic, that the density is uniform in solid and liquid, and that there is no convection in the liquid. In our case of solidification with boundary-interface intersection, the associated free energy functional is assumed to be of the Ginzburg-Landau form with an additional surface energy term in order to account for the boundaryinterface interaction[3]:

$$
\mathcal{F}(\varphi, u)=\int_{\Omega}\left[\frac{1}{2} \varepsilon|\nabla \varphi|^{2}+\varepsilon^{-1} f(\varphi, u)\right] d \mathbf{x}+\int_{\partial \Omega} \gamma(\varphi) d A,
$$

where the small parameter $\varepsilon$ is gradient energy coefficient which also characterize the thickness of the interface, $f(\varphi, u)$ is the bulk free energy density in $\Omega$ and $\gamma(\varphi)$ is the surface free energy density on the external boundary. The square-gradient term is the contribution of the interface. The integrations are understood as in some suitable finite domain under consideration.

By variational principle, the body chemical potential $\mu$ and surface chemical potential $G$ are given by

$$
\mu=-\varepsilon \triangle \varphi+\varepsilon^{-1} \partial f / \partial \varphi \text { and } G=\varepsilon \partial \varphi / \partial n+\gamma^{\prime}(\varphi)
$$


to ensure that the free energy decreases with time, the dynamical equation for $\varphi$ can be given by

$$
\varepsilon \frac{\tau}{\delta} \varphi_{t}=\varepsilon \triangle \varphi-\varepsilon^{-1} \partial f / \partial \varphi \text { in } \Omega
$$

with the relaxational boundary condition $[14,15]$

$$
\varepsilon \frac{\eta}{\delta} \varphi_{t}=-\left(\varepsilon \partial \varphi / \partial n+\gamma^{\prime}(\varphi)\right) \text { on } \partial \Omega,
$$

where $n$ denotes the outward normal of $\partial \Omega$. The constants $\tau$ and $\eta$ are the mobilities on the interface and external boundary, respectively, $\delta$ is the interfacial energy per unit area.

To specify the free energy density, it can be worthwhile to keep $\varphi$ fixed in the bulk phase so that the latent heat is released at the interface only. Thus we take the free energy density as

$$
f(\varphi, u)=\frac{1}{2} g(\varphi)-\lambda p(\varphi) u
$$

here $g(\varphi)=\varphi^{2}(\varphi-1)^{2}$ is a double well potential, the conventional dimensionless parameter $\lambda$ is the ratio of the interfacial width to capillary length and $p(\varphi)$ is a smooth function of $\varphi$ that monotonically increase from $p(0)=0$ to $p(1)=1$ in order to incorporate the release of latent heat as solidification occurs. A simple form of $p$ would be $p(\varphi)=\varphi^{2}(3-2 \varphi)$.

The equation of heat diffusion is modified by incorporating time rate of change of the order parameter $\varphi$ to take into account the latent heat released at the interface, thus

$$
u_{t}+r^{\prime}(\varphi) \varphi_{t}=\nabla \cdot(k(\varphi) \nabla u)
$$

where the function $k(\varphi)$ permits different thermal conductivities $k_{-}=k(0)$ and $k_{+}=k(1)$ in the bulk solid and liquid phases, respectively. The function $r(\varphi)$ is assumed to satisfy $r(0)=0$ and $r(1)=1$. Examples includes the cases $r(\varphi)=p(\varphi)$ and $r(\varphi)=\varphi$, which are referred as the gradient equations and the nongradient equations, respectively, according to Almgren[1]. To summarize, we have the phase field model

$$
\left\{\begin{array}{l}
\varepsilon^{2} \tau \varphi_{t}=\varepsilon^{2} \triangle \varphi-\frac{1}{2} g^{\prime}(\varphi)+\lambda p^{\prime}(\varphi) u \\
u_{t}+r^{\prime}(\varphi) \varphi_{t}=\nabla \cdot(k(\varphi) \nabla u)
\end{array}\right.
$$

with the relaxational boundary condition (7). This problem may be solved by properly imposed initial conditions and boundary condition for $u$.

An asymptotic analysis for $\varepsilon \ll 1$ will be carried out for the solutions of (9), whereby an outer solution, valid away from the interface, is matched to an inner solution that is valid in the interfacial region[5].

For $\varepsilon>0$, we define the interface $\Gamma(t ; \varepsilon)$ to be the level set $\{\varphi=1 / 2\}$. Our primary attention is on the motion of the interface and its intersection with external boundary. Let us parameterize $\Gamma(t ; \varepsilon)$ by $s$. In a neighborhood of $\Gamma(t ; \varepsilon)$, we defined a signed distance function $d(\mathbf{x}, t ; \varepsilon)$ with the same sign as $\varphi(\mathbf{x}, t ; \varepsilon)$. We extend $s$ to this neighborhood by giving $s(\mathbf{x}, t ; \varepsilon)$ its value at the projection point of $\mathbf{x}$ on $\Gamma(t ; \varepsilon)$. Then $(d, s)$ form a local orthogonal curvilinear coordinate system near $\Gamma(t ; \varepsilon)$, satisfying $|\nabla d|=1, \nabla d \cdot \nabla s=0$.

The inner expansion near a point is described via a stretched variable $\rho=d / \varepsilon$. With a slight abuse of notation, for each $\varepsilon$, the functions $\varphi$ and $u$ may be viewed as functions either of $(\mathbf{x}, t)$ or of $(\rho, s, t)$. Elementary computations gives us the formula for converting derivatives over $(\mathbf{x}, t)$ to derivatives over $(\rho, s, t)$ :

$$
\begin{aligned}
& \partial_{x}=\varepsilon^{-1} d_{x} \partial_{\rho}+O(1), \quad \partial_{y}=\varepsilon^{-1} d_{y} \partial_{\rho}+O(1), \\
& \triangle=\varepsilon^{-2} \partial_{\rho \rho}+\varepsilon^{-1} \triangle d \partial_{\rho}+O(1), \\
& \partial_{t}=\varepsilon^{-1} d_{t} \partial_{\rho}+O(1) .
\end{aligned}
$$

Notice that $\nabla d=\left(d_{x}, d_{y}\right),|\nabla d|=1 . \triangle d=\kappa$ is the curvature of the level surface of $d$, normal translations of $\Gamma . d_{t}=-V$ is the negative normal velocity of the interface. 


\section{The outer expansions}

We firstly consider the outer expansion away from the interface

$$
\begin{aligned}
u(\mathbf{x}, t ; \varepsilon) & =u^{0}(\mathbf{x}, t)+\varepsilon u^{1}(\mathbf{x}, t)+O\left(\varepsilon^{2}\right), \\
\varphi(\mathbf{x}, t ; \varepsilon) & =\varphi^{0}(\mathbf{x}, t)+\varepsilon \varphi^{1}(\mathbf{x}, t)+O\left(\varepsilon^{2}\right),
\end{aligned}
$$

with

$$
\varphi^{0}=\left\{\begin{array}{ll}
0 & \text { solid, } \\
1 & \text { liquid, }
\end{array} \quad \varphi^{i} \equiv 0 \text { to all orders } i>1,\right.
$$

thus the leading order temperature equations in the outer regions are

$$
u_{t}^{0}=k_{ \pm} \triangle u^{0} \text { in } \Omega_{ \pm} .
$$

To solve the outer problem, we need further to find the equation for the interface $\Gamma$, and the transmission conditions on $\Gamma$. These will be derived from the inner problem near the interface.

\section{The inner expansions}

In the inner region near a point $\mathbf{x}_{0}$ on the interface, we use the stretched variable $\rho=d / \varepsilon$ and expand

$$
\begin{aligned}
& u=U(\rho, s, t ; \varepsilon)=U^{0}(\rho, s, t)+\varepsilon U^{1}(\rho, s, t)+O\left(\varepsilon^{2}\right), \\
& \varphi=\Phi(\rho, s, t ; \varepsilon)=\Phi^{0}(\rho, s, t)+\varepsilon \Phi^{1}(\rho, s, t)+O\left(\varepsilon^{2}\right),
\end{aligned}
$$

the resulting equations are solved order by order in $\varepsilon$, with far field boundary conditions that are obtained by matching to the outer solutions. This requires the matching conditions

$$
\lim _{\rho \rightarrow \pm \infty} U^{0}(\rho, s, t)=u_{ \pm}^{0}\left(\mathbf{x}_{0}\right),
$$

and

$$
U^{1}(\rho, s, t) \sim \partial_{d} u_{ \pm}^{0}\left(\mathbf{x}_{0}\right) \rho+u_{ \pm}^{1}\left(\mathbf{x}_{0}\right) \text { as } \rho \rightarrow \pm \infty
$$

For $\varphi$, we have

$$
\lim _{\rho \rightarrow \pm \infty} \Phi^{0}(\rho, s, t)=1,0, \text { respectively; } \quad \lim _{\rho \rightarrow \pm \infty} \Phi^{i}(\rho, s, t)=0, j \geq 1 .
$$

By our definition that $\varphi=1 / 2$ on $\Gamma$, we also have the condition that

$$
\Phi^{0}(0, s, t)=1 / 2 ; \quad \Phi^{i}(0, s, t)=0, \quad j \geq 1 .
$$

\section{Classical asymptotics}

We reformulate (9) in curvilinear coordinates $(d, s, t)$ and rescale $d$ to $\rho=d / \varepsilon$, by using the differential relations (10), to obtain

$$
\begin{aligned}
& -\varepsilon \frac{\tau}{\delta} V \Phi_{\rho}=\Phi_{\rho \rho}+\varepsilon \kappa \Phi_{\rho}-\frac{1}{2} g^{\prime}(\Phi)+\lambda p^{\prime}(\Phi) U+O\left(\varepsilon^{2}\right), \\
& -\varepsilon^{-1} V\left(U_{\rho}+r^{\prime}(\Phi) \Phi_{\rho}\right)=\varepsilon^{-2}\left(k(\Phi) U_{\rho}\right)_{\rho}+\varepsilon^{-1} \kappa k(\Phi) U_{\rho}+O(1) .
\end{aligned}
$$

A conventional model with $\lambda=\varepsilon \Lambda$ assumed to be a small parameter will be considered. This requires the interface width assumed to be small compared to the capillary length. This requirement however can be loosen by "thin interface model" [8,9] or "isothermal asymptotics"[1], in which $\lambda$ is assumed to be of order unity but $U^{0}=0$ so that $U=O(\varepsilon)$ and $\lambda U=O(\varepsilon)$ is still retained, see [12]. We now 
substitute the inner expansion (14) into (15) and use the matching conditions to get the structure and motion of the interface.

1. Leading order phase field equation

$$
\Phi_{\rho \rho}^{0}-\frac{1}{2} g^{\prime}\left(\Phi^{0}\right)=0
$$

the solution is

$$
\Phi^{0}(\rho)=\frac{1}{2}\left(\tanh \frac{\rho}{2}+1\right),
$$

which is monotone increasing and approaches its limiting value exponentially as $\rho \rightarrow \pm \infty$.

2. The leading order temperature equation is

$$
\left(k(\Phi) U_{\rho}\right)_{\rho}=0,
$$

integrating and using the matching condition to get

$$
U^{0}=u_{+}^{0}\left(\mathbf{x}_{0}\right)=u_{-}^{0}\left(\mathbf{x}_{0}\right)=\text { constant } .
$$

3. First order phase field equation

$$
\Phi_{\rho \rho}^{1}-\frac{1}{2} g^{\prime \prime}\left(\Phi^{0}\right) \Phi^{1}=-\Lambda U^{0} p^{\prime}\left(\Phi^{0}\right)-\left(\frac{\tau}{\delta} V+\kappa\right) \Phi_{\rho}^{0} .
$$

The solvability condition yields

$$
0=\int\left(\Phi_{\rho \rho}^{1}-\frac{1}{2} g^{\prime \prime}\left(\Phi^{0}\right) \Phi^{1}\right) \Phi_{\rho}^{0} d \rho=-\Lambda U^{0} \int p^{\prime}\left(\Phi^{0}\right) \Phi_{\rho}^{0} d \rho-\left(\frac{\tau}{\delta} V+\kappa\right) \int\left(\Phi_{\rho}^{0}\right)^{2} d \rho,
$$

by denoting $\delta=\int\left(\Phi_{\rho}^{0}\right)^{2} d \rho$ as the interfacial energy, and dimensionless the interfacial temperature $u^{0}$ by $u^{0}\left(\mathbf{x}_{\mathbf{0}}\right)=\Lambda U^{0}$, the above equation leads to

$$
u^{0}\left(\mathbf{x}_{\mathbf{0}}\right)=-\delta \kappa-\tau V
$$

which recovers the Gibbs-Thomson condition (3).

4. First order temperature equation

$$
\left(k\left(\Phi^{0}\right) U_{\rho}^{1}\right)_{\rho}=-V\left(U_{\rho}^{0}+r^{\prime}\left(\Phi^{0}\right) \Phi_{\rho}^{0}\right)-\kappa k\left(\Phi^{0}\right) U_{\rho}^{0} .
$$

Notice that $U^{0}$ is constant thus $U_{\rho}^{0}$ vanishes, integrating once the above equation we have

$$
k\left(\Phi^{0}\right) U_{\rho}^{1}=-\operatorname{Vr}\left(\Phi^{0}\right)+c_{1}(s, t) .
$$

Further integrate to get

$$
U^{1}=-V \int_{0}^{\rho} \frac{r\left(\Phi^{0}\right)}{k\left(\Phi^{0}\right)} d \sigma+c_{1}(s, t) \int_{0}^{\rho} \frac{1}{k\left(\Phi^{0}\right)} d \sigma+c_{2}(s, t),
$$

the integration constants $c_{1}$ and $c_{2}$ are to be determined by matching to the outer solution. For this, we use the far-field expansion

$$
\begin{aligned}
U^{1}(\rho) \sim & \left(\frac{\mp V r_{ \pm}}{k_{ \pm}}+\frac{c_{1}(s, t)}{k_{ \pm}}\right) \rho-V \int_{0}^{\rho}\left(\frac{r\left(\Phi^{0}\right)}{k\left(\Phi^{0}\right)} \mp \frac{r_{ \pm}}{k_{ \pm}}\right) d \sigma \\
& +c_{1}(s, t) \int_{0}^{\rho}\left(\frac{1}{k\left(\Phi^{0}\right)}-\frac{1}{k_{ \pm}}\right) d \sigma+c_{2}(s, t), \text { for } \pm \rho>0,
\end{aligned}
$$


here $r_{ \pm}=r\left(\Phi^{0}( \pm \infty)\right)=1,0$, respectively. By the matching condition $U^{1}(\rho) \sim \partial_{d} u_{ \pm}^{0} \rho+u_{ \pm}^{1}$ as $\rho \rightarrow \pm \infty$, we have

$$
\begin{aligned}
& \partial_{d} u_{ \pm}^{0}=\frac{\mp V r_{ \pm}}{k_{ \pm}}+\frac{c_{1}(s, t)}{k_{ \pm}} \\
& u_{ \pm}^{1}=-V \int_{0}^{ \pm \infty}\left(\frac{r\left(\Phi^{0}\right)}{k\left(\Phi^{0}\right)} \mp \frac{r_{ \pm}}{k_{ \pm}}\right) d \sigma+c_{1}(s, t) \int_{0}^{ \pm \infty}\left(\frac{1}{k\left(\Phi^{0}\right)}-\frac{1}{k_{ \pm}}\right) d \sigma+c_{2}(s, t),
\end{aligned}
$$

then

$$
-V=\left[\left[k \partial_{d} u^{0}\right]\right]_{-}^{+}
$$

which is the jump condition (2).

5. Boundary-interface intersection. Now let us derive the boundary-interface intersection condition for $\Gamma$ at $\{y=0, L\}$. The condition for $\Gamma$ at $\{y=L\}$ can be similarly derived. We only consider the contact point at $\{y=0\}$. Take the inner expansion of the relaxational boundary condition (7), we obtain from the leading order that

$$
\frac{\eta}{\delta} V \Phi_{\rho}^{0}=\cos \beta \Phi_{\rho}^{0}+\gamma^{\prime}\left(\Phi^{0}\right)
$$

where $\beta$ is the dynamic contact angle between the sharp interface $\Gamma$ and the $x$-axis. Multiply the above equation by $\Phi_{\rho}^{0}$ and integrate in $\rho$ over $(-\infty,+\infty)$, notice the interfacial energy $\delta=\int\left(\Phi_{\rho}^{0}\right)^{2} d \rho$, then we have

$$
\eta V=\delta \cos \beta+[[\gamma]]_{-}^{+}
$$

\section{The leading order behavior}

We now summarize the results obtained from the above asymptotic analysis. The leading profiles of the outer solutions $u_{ \pm}^{0}(\mathbf{x}, t)$ satisfy the heat equation

$$
\partial_{t} u_{ \pm}^{0}=k_{ \pm} \triangle u_{ \pm}^{0} \text { in } \Omega_{ \pm}
$$

with a free boundary $\Gamma(t)$ and a jump condition

$$
\left[\left[k \partial_{d} u^{0}\right]\right]_{-}^{+}=-V \text { across } \Gamma(t)
$$

where $V$ is the normal velocity of the evolution of the interface determined by the kinetic relation (see from Gibbs-Thomson condition):

$$
-\tau V=u^{0}\left(\mathbf{x}_{\mathbf{0}}\right)+\delta \kappa \text { on } \Gamma(t)
$$

this dynamics of the interface is constrained by

$$
\eta V=\delta \cos \beta+[[\gamma]]_{-}^{+}
$$

at the the boundary-interface contact point $\Gamma \cap\{y=0\}$ and a similar condition at $\Gamma \cap\{y=L\}$. Here $\beta$ is the dynamic contact angle between the interface $\Gamma$ and the $x$-axis. The Young's equation is recovered by introducing an "equilibrium contact angle" $\beta_{0}$ which is defined when the contact point velocity $V=0$. The problem (21)-(24) may be completely solved by further specified initial and boundary conditions(on physical boundary). 


\section{Conclusion}

In this paper a phase field model for solidification with boundary interface intersection is developed. To balance the interface energy and boundary surface energy in the multiphase contact region, a surface free energy term is incorporated in the free energy functional [3], and a relaxation boundary condition $[14,15]$ for the phase field variable is introduced. By asymptotic analysis, the leading order free interface problem is derived with constraint at the contact point to accommodate the dynamic nature of the contact angle. This problem may be completely solved by further specified initial conditions and boundary conditions on physical boundary.

\section{Acknowledgments}

This research is inspired by Professor Xiao-Ping Wang. The author would like to thank his kind invitation to visit The Hong Kong University of Science and Technology. This research is supported in part by the Fundamental Research Funds for the Central Universities and National Natural Science Foundation of China(Nos.11126082 and 11301182).

\section{References}

[1] R.F. Almgren, Second-order phase field asymptotics for unequal conductivities, SIAM J. Appl. Math. 59(6)(1999) 2086-2107.

[2] G. Caginalp, An analysis of a phase field model of a free boundary, Arch. Rat. Mech. Anal. 92 (1986) 205-245.

[3] J.W. Cahn, Critical point wetting, J. Chem. Phys. 66 (1977) 3667-3672.

[4] S.I. Ei, M.H. Sato, E. Yanagida, Stability of Stationary Interfaces with Contact Angle in a Generalized Mean Curvature Flow, American Journal of Mathematics. 118(3) (1996) 653-687.

[5] P.C. Fife, Dynamics of internal layers and diffusive interfaces, SIAM, Philadephia, PA, 1988.

[6] G. Fix, Phase field methods for free boundary problems, in: Free Boundary Problems, A. Fasano and M. Primicerio, eds., Pitman, London, 1983, pp. 580-589.

[7] B.I. Halperin, P.C. Hohenberg, S. Ma, Renormalization Group Methods for Critical Dynamics: I. Recursion Relations and Effects of Energy Conservation, Phys. Rev. B. 10 (1974) 139-153.

[8] A. Karma, W.J. Rappel, Phase-field method for computationally efficient modeling of solidification with arbitrary interface kinetics, Phys. Rev. E. 53 (1996) R3017-R3020.

[9] A. Karma, W. J. Rappel, Quantitative phase-field modeling of dendritic growth in two and three dimensions, Phys. Rev. E. 57 (1998) 4323-4349.

[10] M. Katsoulakis, G. T. Kossioris, F. Reitich, Generalized Motion by Mean Curvature with Neumann Conditions and the Allen-Cahn Model for Phase Transitions, The Journal of Geometric Analysis, 5(2)(1995) 255-279.

[11] J.S. Langer, Models of pattern formation in first-order phase transitions, in: Directions in Condensed Matter Physics, World Science Publishers, 1986, pp. 164-186.

[12] G.B. McFadden, A.A. Wheeler, D. M. Anderson, Thin interface asymptotics for an energy/entropy approach to phase-field models with unequal conductivities, Physica D. 144 (2000) 154-168. 
[13] M. Plapp, Remarks on some open problems in phase-field modelling of solidification, Philosophical Magazine. 91 (2011) 25-44.

[14] T.Z. Qian, X.P. Wang, P. Sheng, Generalized Navier boundary condition for the moving contact line, Comm. Math. Sci. 1 (2003) 333-341.

[15] T.Z. Qian, X.P. Wang, P. Sheng, Molecular scale contact line hydrodynamics of immiscible flows, Physical Review E. 68 (2003) 016306.

[16] N.K. Simha, K. Bhattacharya, Edge effects on the propagation of phase boundaries, Materials Science and Engineering A. 273-275 (1999) 241-244.

[17] I. Singer-Loginova, H.M. Singer, The phase field technique for modeling multiphase materials, Rep. Prog. Phys. 71 (2008) 106501. 